Pacific Journal of Mathematics

BIJECTIVE PROOFS OF BASIC HYPERGEOMETRIC SERIES 


\title{
BIJECTIVE PROOFS OF BASIC HYPERGEOMETRIC SERIES IDENTITIES
}

\section{J. T. Joichi AND Dennis Stanton}

\begin{abstract}
Bijections are given which prove the following theorems: the $q$-binomial theorem, Heine's ${ }_{2} \Phi_{1}$ transformation, the $q$-analogues of Gauss', Kummer's, and Saalschütz's theorems, the very well poised ${ }_{4} \Phi_{3}$ and ${ }_{6} \Phi_{5}$ evaluations, and Watson's transformation of an ${ }_{8} \Phi_{7}$ to $a_{4} \Phi_{3}$. The proofs hold for all values of the parameters. Bijective proofs of the terminating cases follow from the general case. A bijective version of limiting cases of these series is also given. The technique is to mimic the classical proofs, based upon a bijective proof of the $q$-binomial theorem and sign-reversing involutions which cancel infinite products.
\end{abstract}

1. Introduction. In 1969 George Andrews [1] began to develop a calculus for partition functions. His stated goal was to "...translate a sizable portion of the techniques of the elementary theory of basic hypergeometric series into arithmetic terms". Ideally, he wanted to prove any theorem in basic hypergeometric series by a bijection. In this paper we show (under certain requirements) that this can be accomplished.

Andrews' main object was to give bijective proofs of partition theorems (such as the Rogers-Ramanujan identities). It was well-known that these theorems were closely related to basic hypergeometric series. If a bijective proof of a partition theorem were desired, could one possibly give a bijective proof of a related basic hypergeometric series? If each step in the manipulation of a basic hypergeometric series could be interpreted bijectively, the result would be a bijective proof of the partition theorem.

Andrews gave a bijective proof of the $q$-binomial theorem, which is the cornerstone of basic hypergeometric series. He also showed how to combinatorially interpret cancellations of infinite products, a manipulation of basic hypergeometric series which occurs frequently. However, he used the principle of inclusion-exclusion which, strictly speaking, is not bijective because it cancels objects in clumps. From the recent work of Gessel-Viennot [13] and Garsia-Milne [11] the appropriate bijective replacement for the principle of inclusion-exclusion is a sign-reversing involution. So this part of the theory of basic hypergeometric series can now be done bijectively, which is the main purpose of this paper. 
Initially, we adopt the following convention for a bijective proof of a series identity. To show that $F(q)=G(q)$, we must find two sets, $A$ and $B$, with weights $\mathrm{Wt}(A)$ and $\mathrm{Wt}(B)$, such that

$$
F(q)=\sum_{a \in A} \mathrm{Wt}(a) \text { and } G(q)=\sum_{b \in B} \mathrm{Wt}(b),
$$

and a bijection $\varphi: A \rightarrow B$ which preserves the weight, $\mathrm{Wt}(\varphi(a))=\mathrm{Wt}(a)$. The sets $A$ and $B$ can be infinite. In this case we assume that $A$ and $B$ are countable unions of finite subsets and that $\varphi$ is a bijection between these finite sets. Thus, the identity $F(q)=G(q)$ takes place in the appropriate power series ring. A modification of this convention will be given in $\S 3$.

In order for the bijective proofs to be sufficiently general, our bijections must satisfy certain requirements; we list these requirements here.

$\mathrm{R}_{1}$ If an identity contains parameters besides $q$, the bijection should hold for all values of these parameters. No analytic continuation will be allowed.

$\mathbf{R}_{2}$ Any series manipulation should correspond to a manipulation with bijections.

$R_{3} \quad$ Any cancellation of infinite products should be bijective.

$\mathbf{R}_{4}$ The analytic process of taking limits should correspond to a restriction of a bijection and therefore, also be bijective.

$\mathrm{R}_{5}$ The combinatorial meaning of a specialization of a parameter should be apparent.

For $\mathbf{R}_{1}$, note that there has been much previous work on bijections and generating functions, e.g., [10], [15] or [19]. For basic hypergeometric series there are bijective proofs for special choices of the parameters [9], [10], [14] or [20] which then use analytic continuation. Requirement $R_{2}$ is precisely the program of Garsia-Milne [12] for the Rogers-Ramanujan identities. They used $\mathrm{R}_{3}$ also. Partition theorems ([5], [16]) naturally follow by taking limits of basic hypergeometric series, so we need $R_{4}$. It is clear that $\mathrm{R}_{5}$ is desirable.

The rest of the paper is organized in the following way. Some notation and terminology are in $\S 2$. In $\S 3$ we discuss involutions and the Involution Principle. Our two basic bijections $\Phi$ and $\Psi$ are proven in $\S 4$. We use our bijection $\Phi$ to prove various forms of the $q$-binomial theorem in $\S 5$ and our bijection $\Psi$ is used in $\S 6$ to prove various ${ }_{2} \Phi_{1}$ results. In $\S 5$ and later, theorems are stated simply as identities; it will be understood that what is intended is that there exists a weight preserving signed 
bijection (defined in §3) between two sets whose weights are given by the expressions in the identity. Also, as indicated above, any unrestricted parameters appearing in the expressions may be thought of as indeterminates and that the identity is valid in the appropriate power series ring. We sketch bijective proofs of transformations of higher basic hypergeometric series in $\$ 7$ and conclude with some remarks in $\S 8$.

2. Some notation and terminology. For a partition $\lambda$ of an integer $n$, let $|\lambda|=$ number of parts and $\|\lambda\|=n=$ sum of parts of $\lambda$. Although partitions are usually restricted to positive parts, we not only allow 0 but also, on occasions, negative parts. We define the weight of a partition $\lambda$ by $\mathrm{Wt}(\lambda)=q^{\|\lambda\|}$ and for a parameter $x, \operatorname{Wt}(\lambda ; x)=q^{\|\lambda\|} x^{|\lambda|}$. We let $\varnothing$ denote the empty partition, i.e., the partition with no parts; its weight will always be $\operatorname{Wt}(\varnothing)=1$. If $S$ denotes a set of partitions, then we define $\mathrm{Wt}(S)=\sum_{\lambda \in S} \mathrm{Wt}(\lambda)$ and $\mathrm{Wt}(S(x))=\sum_{\lambda \in S} \mathrm{Wt}(\lambda ; x)$. We shall often refer to the "set" $S(x)$. For sets $S(x)$ and $T(y), \mathrm{Wt}(S(x) \times T(y))=$ $\mathrm{Wt}(S(x)) \cdot \mathrm{Wt}(T(y))$; similarly for more than two sets. A mapping $\varphi$ from $S(x)$ to $T(x)$ will be called a weight-preserving (WP) bijection if (i) $\varphi$ is a bijection from $S$ onto $T$, and (ii) for each $\lambda \in S$, $\mathrm{Wt}(\varphi(\lambda) ; x)=\mathrm{Wt}(\lambda ; x)$. A WP-bijection between products of sets of partitions is defined similarly.

The sets of all partitions, partitions into even parts, partitions into odd parts and partitions into distinct parts will be denoted by $P, P E, P O$ and $P D$, respectively. Also, combinations of these symbols will be used; e.g., $P E D=$ partitions into even distinct parts. If $S$ is a set of partitions, $I$ is an interval of integers and $k$ is a non-negative integer, then $S_{k}^{I}$ denotes the subset of $S$ consisting of those partitions with exactly $k$ parts and each part in the interval $I$. The absence of a subscript indicates that the number of parts is unrestricted. With these conventions, we have

$$
\begin{aligned}
(a)_{n} & =(a ; q)_{n}=(1-a)(1-a q) \cdots\left(1-a q^{n-1}\right) \\
& =\mathrm{Wt}\left(P D^{[0, n-1]}(-a)\right) ;
\end{aligned}
$$

note that the part of size 0 corresponds to the term $-a$ in the first factor.

$$
\begin{gathered}
(a)_{\infty}=(1-a)(1-a q)\left(1-a q^{2}\right) \cdots=\mathrm{Wt}\left(P D^{[0, \infty)}(-a)\right) \\
1 /(b)_{n}=\mathrm{Wt}\left(P^{[0, n-1]}(b)\right) \\
1 /(b)_{\infty}=\mathrm{Wt}\left(P^{[0, \infty)}(b)\right) .
\end{gathered}
$$

Also, we shall use the standard notation for basic hypergeometric series as in [5] or [16]. 
Let $S=\bigcup_{k=0}^{\infty} P_{k}^{[1, n]}$ and let $S^{*}$ denote the set of conjugates of the partitions in $S$; that is, $S^{*}=\bigcup_{k=0}^{n} P_{k}^{[1, \infty)}$. For each $\lambda^{*} \in S$ let $\lambda^{\prime}$ be $\lambda^{*}$ with enough parts of size 0 adjoined to make a total of $n$ parts and let $S^{\prime}=\left\{\lambda^{\prime} \mid \lambda^{*} \in S^{*}\right\}=P_{n}^{[0, \infty)}$. Since there exists a bijection between the sets $S$ and $S^{\prime}$ and $1 /(q)_{n}=\mathrm{Wt}(S)$, it follows that

$$
\frac{x^{n}}{(q)_{n}}=\mathrm{Wt}\left(P_{n}^{[0, \infty)}(x)\right) \text {. }
$$

3. Involutions and the involution principle. In this section, we consider some sign-reversing weight-preserving (SRWP) involutions and other related matters which appear in later sections. These involutions were first given by Garsia-Milne [11]. The SRWP-involutions correspond to cancellations of products; e.g.,

$$
\begin{gathered}
\frac{(x)_{\infty}}{(x)_{\infty}}=1 \\
\frac{(x)_{\infty}}{(x)_{n}}=\left(x q^{n}\right)_{\infty} \text { and } \quad \frac{(x)_{n}}{(x)_{\infty}}=\frac{1}{\left(x q^{n}\right)_{\infty}} \\
\frac{(x)_{\infty}}{\left(x q^{n}\right)_{\infty}}=(x)_{n} \quad \text { and } \quad \frac{\left(x q^{n}\right)_{\infty}}{(x)_{\infty}}=\frac{1}{(x)_{n}} .
\end{gathered}
$$

To prove the first cancellation in (3.2), we need to produce a SRWPinvolution $\theta$ in $P D^{[0, \infty)}(-x) \times P^{[0, n-1]}(x)$ with fixed point set $P D^{[n, \infty)}(-x) \times\{\varnothing\}$; i.e., for any $(\lambda, \mu) \in P D^{[0, \infty)}(-x) \times P^{[0, n-1]}(x)$, either (i) $(\lambda, \mu)$ is a fixed point of $\theta$, or (ii) if $\left(\lambda^{\prime}, \mu^{\prime}\right)=\theta(\lambda, \mu) \neq(\lambda, \mu)$, then $\mathrm{Wt}\left(\lambda^{\prime} ;-x\right) \cdot \mathrm{Wt}\left(\mu^{\prime} ; x\right)=-\mathrm{Wt}(\lambda ;-x) \cdot \mathrm{Wt}(\mu ; x)$. It will follow that

$$
\begin{aligned}
\frac{(x)_{\infty}}{(x)_{n}} & =\mathrm{Wt}\left(P D^{[0, \infty)}(-x) \times P^{[0, n-1]}(x)\right) \\
& =\mathrm{Wt}\left(P D^{[n, \infty)}(-x)\right)=\left(x q^{n}\right)_{\infty} .
\end{aligned}
$$

We define $\theta$ as follows,

(i) if the smallest part of $\lambda$ is greater than or equal to $n$ and $\mu=\varnothing$, then $\theta(\lambda, \mu)=(\lambda, \mu)$, and otherwise

(ii) let $\alpha$ be the largest part of $\lambda$ or $\mu$ which is less than $n$; if $\alpha$ is a part of $\lambda$, then delete it from $\lambda$ and adjoin it to $\mu$, if $\alpha$ is not a part of $\lambda$, then delete it from $\mu$ (once) and adjoin it to $\lambda$.

It is clear that $\theta$ has the desired properties and that we can find SRWP-involutions for the other cancellations in a similar manner, including the cases where we replace $x$ by $x q^{k}$. 
In later sections we shall also need the following identities.

$$
\begin{array}{cc}
(-a ; q)_{n}(a ; q)_{n}=\left(a^{2} ; q^{2}\right)_{n} & 0<n \leq \infty \\
\frac{1}{(-a ; q)_{n}} \frac{1}{(a ; q)_{n}}=\frac{1}{\left(a^{2} ; q^{2}\right)_{n}} & 0<n \leq \infty \\
\frac{(a ; q)_{\infty}}{\left(a ; q^{2}\right)_{\infty}}=\left(a q ; q^{2}\right)_{\infty} .
\end{array}
$$

For (3.4), let

$$
A=P D^{[0, n-1]}(a) \times P D^{[0, n-1]}(-a) \quad \text { and } \quad B=P E D^{[0,2 n-2]}\left(-a^{2}\right) .
$$

We define a SRWP-involution $\theta$ in $A$ by: for $(\lambda, \mu) \in A$,

(i) if $\lambda=\mu$, then $\theta(\lambda, \mu)=(\lambda, \mu)$, and

(ii) if $\lambda \neq \mu$, let $\alpha$ be the smallest part which belongs to one of but not both $\lambda$ and $\mu$; if $\alpha$ is a part of $\lambda$, delete it and adjoin it to $\mu$; if $\alpha$ is a part of $\mu$, delete it and adjoin it to $\lambda$.

For $(\lambda, \lambda) \in A, \lambda=\left(\lambda_{1}, \ldots, \lambda_{k}\right)$, let $\varphi(\lambda, \lambda)=\left(2 \lambda_{1}, \ldots, 2 \lambda_{k}\right)$; then $\varphi$ is a WP-bijection of the fixed point set of $\theta$ onto $B$.

A SWRP-involution is a special case of a WP-signed bijection. A WP-signed bijection $(\theta, \psi ; \varphi)$ between sets $A$ and $B$ consists of

$\left\{\begin{array}{l}\text { (i) SRWP-involutions } \theta \text { and } \psi \text { in } A \text { and } B, \\ \text { with fixed point sets } A^{\prime} \text { and } B^{\prime} \text {, and } \\ \text { (ii) a WP-bijection } \varphi \text { from } A^{\prime} \text { to } B^{\prime} .\end{array}\right.$

In the case (3.2) discussed above, if we let $A=P D^{[0, \infty)}(-x) \times$ $P^{[0, n-1]}(x), A^{\prime}=P D^{[n, \infty)}(-x) \times\{\varnothing\}, B=B^{\prime}=P D^{[n, \infty)}(-x)$, and let $\psi$ be the identity, then we have a special case of a WP-signed bijection. We use the following notation to describe this case,

$$
A \stackrel{\theta}{\downarrow} B \quad \text { (the bijection } \varphi \text { is suppressed). }
$$

The case $\theta$ is the identity is denoted,

$$
A \stackrel{\psi}{\uparrow} B .
$$

It is clear that a composition of WP-bijections is a WP-bijection. The composition of two WP-signed bijections is again a WP-signed bijection; this is guaranteed by the Involution Principle of Garsia-Milne [11]. In this paper, the finiteness condition for the Involution Principle will always be 
satisfied. This follows since all of our bijections and involutions preserve weights, and the number of objects with a given weight is finite. Thus, all bijections and involutions can be restricted to finite sets.

In later sections we will be concerned with when we need to invoke the Involution Principle. The two following special cases of compositions of WP-signed bijections, will occur frequently. The first case is $(\theta, \psi ; \varphi)$ with $\theta$ the identity followed by $\left(\theta^{\prime}, \psi^{\prime} ; \varphi^{\prime}\right)$ with $\psi^{\prime}$ the identity. Here, the Involution Principle is usually used. However, in some cases, $\theta^{\prime}$ maps the image of $A$

$$
A \uparrow \stackrel{\psi}{\uparrow^{\prime}} \stackrel{\theta^{\prime}}{\downarrow} C
$$

in $B$ onto itself and we have a WP-injection of its fixed point set onto $C$. The second case is $\psi$ and $\theta^{\prime}$ the identity. It is clear that the Involution Principle is not used since the fixed points of $\theta$ can be identified with those of $\psi^{\prime}$ by $\varphi^{\prime} \circ \varphi$,

$$
A \stackrel{\theta}{\downarrow} B \stackrel{\psi^{\prime}}{\uparrow} C
$$

4. The bijections $\Phi$ and $\Psi$. In this section we give the bijections which will prove the $q$-binomial theorem $(\Phi)$ and Heine's transformation $(\Psi)$.

LEMMA 4.1. There exists a WP-bijection $\Phi$ from the set

$$
\bigcup_{n=0}^{\infty} P D^{[0, n-1]}(-a) \times P_{n}^{[0, \infty)}(x)
$$

onto the set

$$
P D^{[0, \infty)}(-a x) \times P^{[0, \infty)}(x) .
$$

In particular, for each $n \geq 0$ and $0 \leq k \leq n$,

$$
\Phi\left(P D_{k}^{[0, n-1]}(-a) \times P_{n}^{[0, \infty)}(x)\right)=P D_{k}^{[0, \infty)}(-a x) \times P_{n-k}^{[0, \infty)}(x) .
$$

Proof. Suppose $\lambda=\left(\lambda_{1}, \lambda_{2}, \ldots, \lambda_{k}\right) \in P D_{k}^{[0, n-1]}(-a)$ where $n-1$ $\geq \lambda_{1}>\lambda_{2}>\cdots>\lambda_{k} \geq 0$ and $\mu=\left(\mu_{1}, \mu_{2}, \ldots, \mu_{n}\right) \in P_{n}^{[0, \infty)}(x)$ where $\mu_{1} \geq \mu_{2} \geq \cdots \geq \mu_{n} \geq 0$. Then $\left(\lambda^{\prime}, \mu^{\prime}\right)=\Phi(\lambda, \mu) \in P D_{k}^{[0, \infty)}(-a x) \times$ $P_{n-k}^{[0, \infty)}(x)$ is obtained by the following algorithm. 


\section{Algorithm $\Phi$}

1. Let $\lambda^{*}$ be the conjugate of $\lambda$ where 0 's have been adjoined to make $n$ parts.

2. Let $\nu$ be the partition obtained by adding corresponding parts of $\lambda^{*}$ and $\mu$.

3. $\lambda^{\prime}$ is obtained from $\nu$ by selecting the $\left(\lambda_{k}+1\right)$ th, $\left(\lambda_{k-1}+1\right)$ th, $\ldots,\left(\lambda_{1}+1\right)$ th parts.

4. $\mu^{\prime}$ consists of what remains of $\nu$.

Clearly, $\Phi$ is one-to-one and weight-preserving. To show that it is onto, we give an algorithm for $\Phi^{-1}$. Suppose $\lambda^{\prime}=\left(\lambda_{1}^{\prime}, \ldots, \lambda_{k}^{\prime}\right) \in$ $P D_{k}^{[0, \infty)}(-a x)$ and $\mu^{\prime}=\left(\mu_{1}^{\prime}, \ldots, \mu_{n-k}^{\prime}\right) \in P_{n-k}^{[0, \infty)}(x)$. Then $(\lambda, \mu)=$ $\Phi^{-1}\left(\lambda^{\prime}, \mu^{\prime}\right)$ is obtained by the following algorithm.

\section{Algorithm $\Phi^{-1}$}

1. Let $\nu=\left(\nu_{1}, \ldots, \nu_{n}\right)$ be the partition obtained by interlacing the parts of $\lambda^{\prime}$ and $\mu^{\prime}$; if $\lambda_{i}^{\prime}=\mu_{j}^{\prime}$ then $\lambda_{i}^{\prime}$ is placed before $\mu_{j}^{\prime}$.

2. Suppose $\nu_{i_{1}}=\lambda_{1}^{\prime}, \ldots, \nu_{i_{k}}=\lambda_{k}^{\prime}$.

3. $\lambda=\left(i_{k}-1, \ldots, i_{1}-1\right)$.

4. $\mu=\left(\mu_{1}, \ldots, \mu_{n}\right)$ where,

$$
\begin{aligned}
& \text { for } j=1, \ldots, i_{1}-1, \quad \mu_{j}=\nu_{j}-k, \\
& \text { for } j=i_{1}, \ldots, i_{2}-1, \quad \mu_{j}=\nu_{j}-(k-1), \ldots, \\
& \text { for } j=i_{k}, \ldots, n, \quad \mu_{j}=\nu_{j} .
\end{aligned}
$$

EXAmple 4.2. Let $n=6, k=4, \lambda=(5,3,2,0)$ and $\mu=(3,3,2,2,2$, $0)$. Then $\lambda^{*}=(3,3,2,1,1,0)$ and by left justifying the juxtaposition of $\lambda^{*}$ and $\mu$ we get $\nu=(6,6,4,3,3,0)$. The parts of $\lambda$ mark the distinct parts for $\lambda^{\prime} ; \lambda_{4}+1=1, \lambda_{3}+1=3, \lambda_{2}+1=4$ and $\lambda_{1}+1=6$ so $\lambda^{\prime}$ consists of the $1 \mathrm{st}, 3 \mathrm{rd}, 4 \mathrm{th}$ and 6 th parts of $\nu$ and $\mu^{\prime}$ consists of what remains; $\lambda^{\prime}=(6,4,3,0)$ and $\mu^{\prime}=(6,3)$.

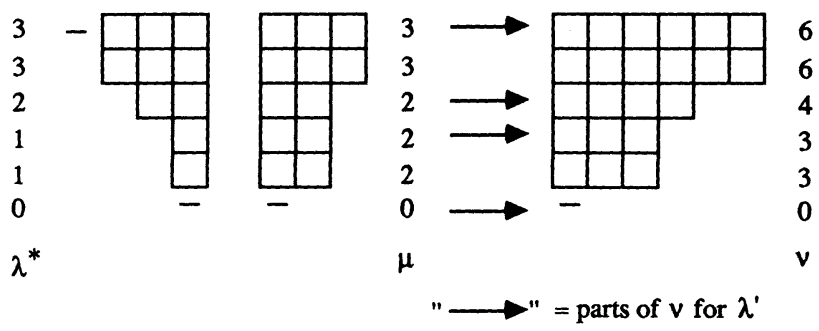


For $\Phi^{-1}$, form $\nu$ from $\lambda^{\prime}$ and $\mu^{\prime}$. For each cell $c$ of $\nu$, count the number of markers $m$ below $c$ and move $c$ to the left $m$ units; the cells which move past the original left edge become cells in $\lambda$ (or $\lambda^{*}$ ) and what remains gives $\mu$. A marker in the first row of $\nu$ corresponds to a 0 part of $\lambda$.

Zeilberger's Algorithm Z [9, p. 226] with $k=\infty$ is a WP-bijection.

$$
P_{s}^{[0, \infty)}(x) \times P_{r}^{[0, s-r]}(a) \stackrel{Z}{\rightarrow} P_{s-r}^{[0, \infty)}(x) \times P_{r}^{[0, \infty)}(a x) .
$$

If we attach parts of size $0,1, \ldots, r-1$ to each partition in $P_{r}$, then $Z$ is a WP-bijection

$$
P_{s}^{[0, \infty)}(x) \times P D_{r}^{[0, s-1]}(a) \stackrel{Z}{\rightarrow} P_{s-r}^{[0, \infty)}(x) \times P D_{r}^{[0, \infty)}(a x) .
$$

However, $Z$ does not coincide with $\Phi$. As we shall see in the next section, this implies that there are two different bijections which proves the $q$-binomial theorem.

The WP-bijection $\Psi$ given below proves Heine's transformation. It is constructed from two applications of $\Phi$, copying the analytic proof in [5, p. 19].

LEMMA 4.5. There exists a WP-bijection $\Psi$ from the set

$$
\bigcup_{n=0}^{\infty} P D^{[0, n-1]}(-a) \times P_{n}^{[0, \infty)}(x) \times P D^{[n, \infty)}(-c) \times P^{[n, \infty)}(b)
$$

onto the set

$$
\bigcup_{m=0}^{\infty} P D^{[m, \infty)}(-a x) \times P^{[m, \infty)}(x) \times P D^{[0, m-1]}\left(-\frac{c}{b}\right) \times P_{m}^{[0, \infty)}(b) .
$$

In particular, for each $n \geq 0,0 \leq k \leq n, m \geq 0$ and $0 \leq j \leq m$, we have $\Psi(L)=R$, where

$$
L=P D_{k}^{[0, n-1]}(-a) \times P_{n}^{[0, \infty)}(x) \times P D_{j}^{[n, \infty)}(-c) \times P_{m-j}^{[n, \infty)}(b)
$$

and

$$
R=P D_{k}^{[m, \infty)}(-a x) \times P_{n-k}^{[m, \infty)}(x) \times P D_{j}^{[0, m-1]}\left(-\frac{c}{b}\right) \times P_{m}^{[0, \infty)}(b) .
$$

Proof. For $(\lambda, \mu, \nu, \xi) \in L,\left(\lambda^{\prime}, \mu^{\prime}, \nu^{\prime}, \xi^{\prime}\right)=\Psi(\lambda, \mu, \nu, \xi)$ is defined by the following algorithm. 


\section{Algorithm $\Psi$}

1. Let $\left(\nu^{\prime \prime}, \xi^{\prime \prime}\right)$ be obtained from $(\nu, \xi)$ by reducing each part by $n$.

2. Let $\left(\lambda^{\prime \prime}, \mu^{\prime \prime}\right)=\Phi(\lambda, \mu)$ and $\left(\nu^{\prime}, \xi^{\prime}\right)=\Phi^{-1}\left(\nu^{\prime \prime}, \xi^{\prime \prime}\right)$.

3. Let $\left(\lambda^{\prime}, \mu^{\prime}\right)$ be obtained from $\left(\lambda^{\prime \prime}, \mu^{\prime \prime}\right)$ by increasing each part by $m$.

5. The $q$-binomial theorem and related identities. In this section we give proofs, using WP-bijections and signed bijections, of several forms of the $q$-binomial theorem and some related identities.

TheOrem 5.1 (The q-Binomial Theorem).

$$
\sum_{n=0}^{\infty} \frac{(a)_{n}}{(q)_{n}} x^{n}=\frac{(a x)_{\infty}}{(x)_{\infty}}
$$

Proof. The identity follows from the existence of our WP-bijection $\Phi$ since

$$
\sum_{n=0}^{\infty} \frac{(a)_{n}}{(q)_{n}} x^{n}=\mathrm{Wt}\left(\bigcup_{n=0}^{\infty} P D^{[0, n-1]}(-a) \times P_{n}^{[0, \infty)}(x)\right)
$$

and

$$
\frac{(a x)_{\infty}}{(x)_{\infty}}=\mathrm{Wt}\left(P D^{[0, \infty)}(-a x) \times P^{[0, \infty)}(x)\right) .
$$

By first reducing part sizes appropriately, applying $\Phi$, and then increasing part sizes, we could have proven the $q$-binomial theorem in the form:

For any integers $M$ and $N, N \geq 0$,

$$
\sum_{n=0}^{\infty} \frac{\left(a q^{M}\right)_{n}}{(q)_{n}}\left(x q^{N}\right)^{n}=\frac{\left(a x q^{M+N}\right)_{\infty}}{\left(x q^{N}\right)_{\infty}}
$$

that is, there exists a WP-bijection from the set

$$
\bigcup_{n=0}^{\infty} P D^{[M, M+n-1]}(-a) \times P_{n}^{[N, \infty)}(x)
$$

onto the set

$$
P D^{[M+N, \infty)}(-a x) \times P^{[N, \infty)}(x) .
$$

THEOREM 5.3 (The $q^{2}$-Binomial Theorem).

$$
\sum_{n=0}^{\infty} \frac{\left(a ; q^{2}\right)_{n}}{\left(q^{2} ; q^{2}\right)_{n}} x^{n}=\frac{\left(a x ; q^{2}\right)_{\infty}}{\left(x ; q^{2}\right)_{\infty}} .
$$


Proof. For $\lambda \in \bigcup_{k=0}^{n} P E D_{k}^{[0,2 n-2]}$, replace each part of $\lambda$ by two parts half as large. Then

$$
\left(a ; q^{2}\right)_{n}=\mathrm{Wt}\left(\bigcup_{k=0}^{n} P_{2 k, 2}^{[0, n-1]}(\sqrt{-a})\right),
$$

where the subscript $2 k, 2$ indicates that of the $2 k$ parts, each distinct part size occurs exactly twice.

The set of conjugates of the partitions in $\bigcup_{k=0}^{\infty} P E_{k}^{[2,2 n]}$ is the set $\bigcup_{k=0}^{n} P_{2 k, E}^{[1, \infty)}$ where the subscript $E$ indicates that each distinct part size occurs an even number of times. Let each pair of equal parts be replaced by one part twice as large and adjoin enough 0 's to make $n$ parts. It follows that

$$
\sum_{n=0}^{\infty} \frac{\left(a ; q^{2}\right)_{n}}{\left(q^{2} ; q^{2}\right)_{n}} x^{n}=\mathrm{Wt}\left(\bigcup_{n=0}^{\infty} \bigcup_{k=0}^{n} P_{2 k, 2}^{[0, n-1]}(\sqrt{-a}) \times P E_{n}^{[0, \infty)}(x)\right) .
$$

For $(\lambda, \mu) \in P_{2 k, 2}^{[0, n-1]}(\sqrt{-a}) \times P E_{n}^{[0, \infty)}(x)$, let $\left(\lambda^{\prime}, \mu^{\prime}\right)$ be determined by Algorithm $\Phi$ modified so that each pair of equal parts of $\lambda$ determines one part of $\lambda^{\prime}$; say $\left(\lambda^{\prime}, \mu^{\prime}\right)=\Phi_{2}(\lambda, \mu)$. Then $\Phi_{2}$ is a WP-bijection onto the set $P E D^{[0, \infty)}(-a x) \times P E^{[0, \infty)}(x)$ as desired.

As before, by a modification of the above, we could have proven the identity in the form:

For any integers $M, N$ and $B$ where $N \geq 0$ and $B>0$,

$$
\sum_{n=0}^{\infty} \frac{\left(a q^{M} ; q^{B}\right)_{n}}{\left(q^{B} ; q^{B}\right)_{n}}\left(x q^{N}\right)^{n}=\frac{\left(a x q^{M+N} ; q^{B}\right)_{\infty}}{\left(x q^{N} ; q^{B}\right)_{\infty}}
$$

Andrews [1] gave a bijective proof of this identity in the special case where $1 \leq M<N=B$. However, his bijection is not defined for $B=1$ or for the argument $x$ (instead of $x q^{B}$ ) of the ${ }_{1} \Phi_{0}$. These cases are used throughout the theory of basic hypergeometric series. Our bijection will do all of these cases, and reduces to Andrews' for $1 \leq M<N=B$.

Two special cases of the $q$-binomial theorem were considered by Euler; (i) $a=0$, and (ii) replace $x$ by $x / a$ and take the limit as $a \rightarrow \infty$. These have well-known bijective proofs; for $a=0$, it is clear that our proof is the same. The limiting case

$$
\sum_{n=0}^{\infty} \frac{(-1)^{n} q^{n(n-1) / 2}}{(q)_{n}} x^{n}=(x)_{\infty}
$$

should satisfy requirement $R_{4}$. 
Proof of (5.5). Let $\tau_{n}$ be the partition $\tau_{n}=(n-1, n-2, \ldots, 1,0) \in$ $P D^{[0, n-1]}$. Then (5.5) follows by restricting our WP-bijection $\Phi$ to the subset $\left\{\tau_{n}\right\}(-1) \times P_{n}^{[0, \infty)}(x)$ of $P D^{[0, n-1]}(-1) \times P_{n}^{[0, \infty)}(x)$ followed by the obvious WP-bijection from $P D^{[0, \infty)}(-x) \times\{\varnothing\}$ onto $P D^{[0, \infty)}(-x)$.

There are two cases in which the infinite products in the $q$-binomial theorem cancel; $a=q^{-M}$ or $a=q^{M}$ for integer $M>0$. In order to meet requirement $R_{5}$, bijective proofs of these cases should follow from Theorem 5.1.

The case $a=q^{-M}$ is

$$
\sum_{n=0}^{M} \frac{\left(q^{-M}\right)_{n}}{(q)_{n}} x^{n}=\left(x q^{-M}\right)_{M}
$$

Proof of (5.6). If we set $a=q^{-M}$ in (5.1), we get

$$
\sum_{n=0}^{\infty} \frac{\left(q^{-M}\right)_{n}}{(q)_{n}} x^{n}=\frac{\left(q^{-M} x\right)_{\infty}}{(x)_{\infty}}
$$

Now, for each $n>M$, we have a WPSR-involution in the set

$$
P D^{[-M,-M+n-1]}(-1) \times P_{n}^{[0, \infty)}(x)
$$

(adjoin or delete a part of size 0 in $\lambda \in P D^{[-M,-M+n-1]}$ ) with no fixed points; this involution corresponds to the identity $\left(q^{-M}\right)_{n} /(q)_{n}=0$ for $n>M$.

Let $\theta_{1}$ be the SRWP-involution in the set

$$
\bigcup_{n=0}^{\infty} P D^{[-M,-M+n-1]}(-1) \times P_{n}^{[0, \infty)}(x)
$$

with fixed point set

$$
\bigcup_{n=0}^{M} P D^{[-M,-M+n-1]}(-1) \times P_{n}^{[0, \infty)}(x)
$$

and let $\theta_{2}$ be the WPSR-involution corresponding to the cancellation

$$
\frac{\left(q^{-M} x\right)_{\infty}}{(x)_{\infty}}=\left(q^{-M} x\right)_{M}
$$


We then have

$$
\begin{aligned}
\bigcup_{n=0}^{M} \bigcup_{k=0}^{n} P & D_{k}^{[-M,-M+n-1]}(-1) \times P_{n}^{[0, \infty)}(x) \\
& \theta_{1} \bigcup_{n=0}^{\infty} \bigcup_{k=0}^{n} P D_{k}^{[-M,-M+n-1]}(-1) \times P_{n}^{[0, \infty)}(x) \\
& \stackrel{\Phi}{\rightarrow} \bigcup_{n=0}^{\infty} \bigcup_{k=0}^{n} P D_{k}^{[-M, \infty)}(-x) \times P_{n-k}^{[0, \infty)}(x) \\
& \theta_{2} \\
& \downarrow \bigcup_{k=0}^{M} P D_{k}^{[-M,-1]}(-x) .
\end{aligned}
$$

By looking at (5.7), it appears that the Involution Principle is being used. However, if we let $\tau_{n}^{-M}=(-M+n-1,-M+n-2, \ldots,-M+$ $1,-M)$ then it can be seen that there is a WP-bijection

$$
\bigcup_{n=0}^{M}\left\{\tau_{n}^{-M}\right\}(-1) \times P_{n}^{[0, M-n]}(x) \rightarrow \bigcup_{k=0}^{M} P D_{k}^{[-M,-1]}(-x)
$$

where

$$
\begin{aligned}
\bigcup_{n=0}^{M}\left\{\tau_{n}^{-M}\right. & (-1) \times P_{n}^{[0, M-n]}(x) \\
& \subset \bigcup_{n=0}^{M} \bigcup_{k=0}^{n} P D_{k}^{[-M,-M+n-1]}(-1) \times P_{n}^{[0, \infty)}(x) .
\end{aligned}
$$

Moreover, $\theta_{2}$ (that is, $\Phi^{-1} \circ \theta_{2} \circ \Phi$ ) maps this latter set onto itself.

Note that (5.8) establishes the identity

$$
\sum_{n=0}^{M}(-1)^{n} q^{-M n+n(n-1) / 2}\left[\begin{array}{c}
M \\
n
\end{array}\right] x^{n}=\left(q^{-M} x\right)_{M}
$$

where, for non-negative integers $H$ and $K$,

$$
\left[\begin{array}{c}
H+K \\
K
\end{array}\right]=\mathrm{Wt}\left(P_{K}^{[0, H]}(1)\right)
$$

is the $q$-binomial coefficient or Gaussian polynomial.

We next consider the case $a=q^{M+1}(M \geq 0)$ from which we will arrive at the alternate definition of the $q$-binomial coefficient

$$
\left[\begin{array}{c}
H+K \\
K
\end{array}\right]=\frac{(q)_{H+K}}{(q)_{H}(q)_{K}} .
$$


Since

$$
\frac{(q)_{M+n}}{(q)_{M}(q)_{n}}=\frac{\left(q^{M+1}\right)_{n}}{(q)_{n}}
$$

the case for $a=q^{M+1}$ can be written

$$
\sum_{n=0}^{\infty} \frac{(q)_{M+n}}{(q)_{M}(q)_{n}} x^{n}=\frac{1}{(x)_{M+1}}
$$

Also, since

$$
\begin{aligned}
\frac{1}{(x)_{M+1}} & =\mathrm{Wt}\left(P^{[0, M]}(x)\right)=\mathrm{Wt}\left(\bigcup_{n=0}^{\infty} P_{n}^{[0, M]}(1) \cdot x^{n}\right) \\
& =\sum_{n=0}^{\infty}\left[\begin{array}{c}
n+M \\
n
\end{array}\right] x^{n},
\end{aligned}
$$

(5.10) follows from (5.11) by considering those partitions whose $x$-weight is $x^{n}$.

Proof of (5.11). If we set $a=q^{M+1}$ in (5.1), we get

$$
\sum_{n=0}^{\infty} \frac{\left(q^{M+1}\right)_{n}}{(q)_{n}} x^{n}=\frac{\left(q^{M+1} x\right)_{\infty}}{(x)_{\infty}} \text {. }
$$

Let $\theta_{1}$ be the involution in the set $P D^{[0, M+n]}(-1) \times P^{[0, M]}(1)$ corresponding to the cancellation $\left((q)_{M+n} /(q)_{M}\right)=\left(q^{M+1}\right)_{n}$ and let $\theta_{2}$ be the involution in the set $P D^{[M+1, \infty)}(-x) \times P^{[0, \infty)}(x)$ corresponding to the cancellation $\left(\left(q^{M+1} x\right)_{\infty} /(x)_{\infty}\right)=1 /(x)_{M+1}$. We then have

$$
\begin{aligned}
\bigcup_{n=0}^{\infty} & \left\{P D^{[0, M+n]}(-1) \times P^{[0, M]}(1)\right\} \times P_{n}^{[0, \infty)}(x) \\
& \theta_{1} \bigcup_{n=0}^{\infty}\left\{\bigcup_{k=0}^{n} P D_{k}^{[M+1, M+n]}(-1)\right\} \times P_{n}^{[0, \infty)}(x) \\
& \stackrel{\Phi}{\rightarrow} \bigcup_{n=0}^{\infty} \bigcup_{k=0}^{n} P D_{k}^{[M+1, \infty)}(-x) \times P_{n-k}^{[0, \infty)}(x) \\
& \theta_{2} \\
& \downarrow \bigcup_{n=0}^{\infty} P_{n}^{[0, M]}(x) .
\end{aligned}
$$

This bijective version of (5.10) can also be derived from an involution of Franklin [18, p. 269]. He had a SRWP-involution on $P_{n}^{[0, \infty)}(1) \times$ $P D^{[M+1, M+k]}(-1), k \leq n$, whose fixed point set was $A \times \varnothing, A=\{\lambda \in$ $\left.P_{n}^{[0, \infty)}(1): \lambda=\left(\lambda_{1}, \ldots, \lambda_{n}\right), \lambda_{1}-\lambda_{k+1} \leq M\right\}$. For $k=n, A=P_{n}^{[0, M]}(1)$, 
so if Franklin's involution is combined with $\theta_{1},(5.10)$ results. We also see that applying an involution following Zeilberger's bijection $Z$ (4.3) for $k=\infty$ would give another proof of (5.10).

6. Heine's transformation and its corollaries. The fundamental transformation for $\mathrm{a}_{2} \Phi_{1}$ is Heine's transformation [5]

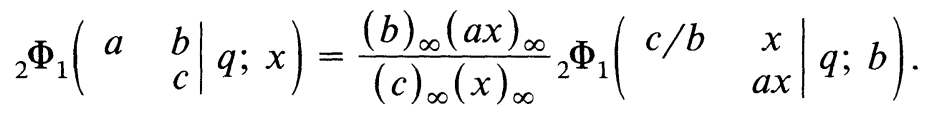

Andrews [2] gave a combinatorial proof of an identity ((6.2)) equivalent to (6.1). Strictly speaking, his proof was not bijective because his WP-map $\varphi$ was defined on disjoint subsets of the appropriate sets $A$ and $B$ and not individual elements. His proof did not correspond to any analytic proof. Also, Andrews did not use his bijective proof of the $q$-binomial theorem [1] to prove any theorems on ${ }_{2} \Phi_{1}$ 's.

The bijection $\Psi$ clearly shows

$$
\sum_{n=0}^{\infty} \frac{(a)_{n}\left(c q^{n}\right)_{\infty}}{(q)_{n}\left(b q^{n}\right)_{\infty}} x^{n}=\sum_{m=0}^{\infty} \frac{(c / b)_{m}\left(a x q^{m}\right)_{\infty}}{(q)_{m}\left(x q^{m}\right)_{\infty}} b^{m} .
$$

The SRWP-involutions corresponding to the cancellations of the infinite products imply

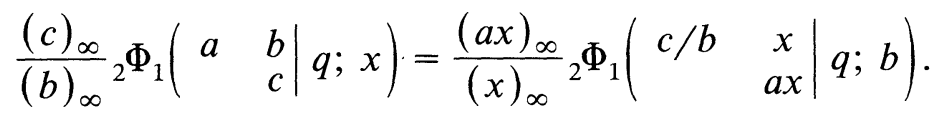

Finally, the SRWP-involutions for $(c)_{\infty} /(c)_{\infty}=(b)_{\infty} /(b)_{\infty}=1$ give (6.1). Note that the Involution Principle has not been used. $\Psi$ is a WP-bijection, so the map for (6.3) is a WP-signed bijection. The fixed points of the left side have been taken.

The first corollary is the $q$-analogue of Gauss' theorem and uses requirement $\mathrm{R}_{5}$

$$
{ }_{2} \Phi_{1}\left(\begin{array}{ll}
a & b \\
& c
\end{array} \mid q ; \frac{c}{a b}\right)=\frac{(c / a)_{\infty}(c / b)_{\infty}}{(c)_{\infty}(c / a b)_{\infty}} .
$$

The analytic proof uses the specialization $x=c / a b$ in Heine's transformation and evaluates the resulting ${ }_{1} \Phi_{0}$ by the $q$-binomial theorem. The bijective version of this is to cancel the term $(c / b)_{n} /(c / b)_{n}$ in the right side sum by an involution, and then use $\Phi$ to bijectively do the $q$-binomial theorem. The result is

$$
{ }_{2} \Phi_{1}\left(\begin{array}{ll}
a & b \\
& c
\end{array} q ; \frac{c}{a b}\right)=\frac{(c / b)_{\infty}(b)_{\infty}(c / a)_{\infty}}{(c / a b)_{\infty}(c)_{\infty}(b)_{\infty}} .
$$


Another involution does the final cancellation to derive (6.4). Here, since we have taken the fixed points of the right side, the Involution Principle has been used. Had we started with (6.3) instead of (6.1), we would not have used the Involution Principle to obtain

$$
\frac{(c)_{\infty}}{(b)_{\infty}} \Phi_{1}\left(\begin{array}{ll}
a & b \\
& c
\end{array} \mid q ; \frac{c}{a b}\right)=\frac{(c / b)_{\infty}(c / a)_{\infty}}{(c / a b)_{\infty}(b)_{\infty}}
$$

Some remarks are in order about the formal power series ring for (6.4), (6.5) and (6.6). The bijection $\Psi$ implied that the coefficient of $q^{n} x^{N} a^{m} b^{k} c^{1}$ on both sides of (6.2) is identical for all $n, N, m, k, l \geq 0$. So (6.2) and (6.1) could take place in any appropriate power series ring. The specialization $x=c / a b$ in (6.1) puts the left side of (6.1) in the ring $Z[1 / a, 1 / b][[c]][[q]]$ and the right in the ring $Z(b)[1 / a][[c]][[q]] \supset$ $Z[1 / a, 1 / b][[c]][[q]]$. The signed bijection shows that the right side is in $Z[1 / a, 1 / b][[c]][[q]]$, so equality takes place in this formal power series ring. Equivalently, we could scale $c$ to $c a b$ and consider formal power series in $q, a, b$ and $c$.

Andrews [3] has given several other applications of Heine's transformation. The $q$-analogue of Kummer's theorem is

$$
\text { (6.7) }{ }_{2} \Phi_{1}\left(\begin{array}{cc}
a & b \\
& q b / a
\end{array} \mid q ;-q / a\right)=\frac{\left(b q ; q^{2}\right)_{\infty}\left(b q^{2} / a^{2} ; q^{2}\right)_{\infty}(-q)_{\infty}}{(b q / a)_{\infty}(-q / a)_{\infty}} \text {. }
$$

Put $c=b q / a$ and $x=-q / a$ in (6.1) and the right side becomes $a_{1} \Phi_{0}$ on base $q^{2}$. The involutions of $\S 3$ do this bijectively. The version of $\Phi$ for the $q^{2}$-binomial Theorem (5.3) does the evaluation. Again, the Involution Principle has been used because we have taken the fixed points of an involution of the right side. The two terminating forms $\left(a=q^{-n}\right.$ and $b=q^{-n}$ ) of Kummer's theorem also follow, by applying involutions as in the proof of (5.6) and (5.11).

In the same way, the $q$-analogues of Bailey's theorem [3, Eq (1.9)] and Gauss' second theorem [3, Eq (1.8)] can be given bijective proofs.

Another consequence of Heine's transformation is the transformation for a terminating Saalschützian (1-balanced) ${ }_{4} \Phi_{3}$. An analytic proof is to first iterate (6.1) three times to obtain the $q$-analogue of Euler's transformation

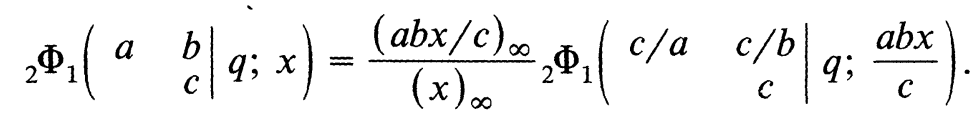

(Note that (6.8) requires 6 applications of $\Phi$ and 23 cancelling involutions.) Next, multiply (6.8) by itself (with parameters $a, b, c ; x$ replaced by $d, e, f ; a b x / c$, and $c f=a d b e)$, and equate coefficients of $x^{n}$ on both 
sides. The bijective version of equating coefficients of $x^{n}$ is restricting a bijection to all partitions whose $x$-weight is $x^{n}$. So we have a bijective proof of

$$
\begin{aligned}
& \sum_{k=0}^{n} \frac{(a)_{k}(b)_{k}(d)_{n-k}(e)_{n-k}}{(q)_{k}(c)_{k}(q)_{n-k}(f)_{n-k}}\left(\frac{a b}{c}\right)^{n-k} \\
& =\sum_{k=0}^{n} \frac{(c / a)_{k}(c / b)_{k}(f / d)_{n-k}(f / e)_{n-k}}{(q)_{k}(c)_{k}(q)_{n-k}(f)_{n-k}}\left(\frac{a b}{c}\right)^{k}, \quad \text { if } c f=\text { abde. }
\end{aligned}
$$

This transformation includes a $q$-analogue of Sheppard's transformation for a ${ }_{3} \Phi_{2}$ and Saalschütz's theorem. Bijective proofs for special choices of the parameters for these two theorems have been given in [9], [14] and [20]. Cancelling involutions allow (6.9) to be written as a ${ }_{4} \Phi_{3}$ transformation.

It is also well-known that ${ }_{3} \Phi_{2}$ transformations follow from Heine's transformation (or the $q$-analogue of Gauss' theorem) by series manipulations. So these theorems also have bijective proofs.

7. Higher transformations. Bijective proofs for higher transformations of basic hypergeometric series can also be given. An analytic way to prove these transformations is to use the Bailey transform (see [7] or [17]), which is a rearrangement of series, on the lower evaluations. The bijective version of the Bailey transform is just rearrangement of unions. This means that we can build successively more complicated bijections to give a bijective proof of Watson's transformation of a ${ }_{8} \Phi_{7}$ to a ${ }_{4} \Phi_{3}$. A short summary of this process is given in the diagram on the following page.

The Rogers-Ramanujan identities follow from a limiting case of Watson's transformation and the Jacobi triple product identity. The limiting case involves $\lim _{b \rightarrow \infty}(a b)_{n} /(b)_{n}=a^{n}$ which corresponds to a restriction of a bijection, as in $\$ 5$. Since the Jacobi triple product identity also has a bijective proof $[8$, p. 4] or $[15$, p. 91$]$, we have, in theory, a bijective proof of the Rogers-Ramanujan identities. It would be quite a task to explicitly give this bijection because the bijective proof of Watson's transformation involves hundreds of involutions and applications of $\Phi$. In fact, the Garsia-Milne proof [12] can be thought of as exactly this process. They began with Schur's analytic proof.

We have not shown that any theorem in basic hypergeometric series has a bijective proof. The theorem in question must have a proof which is a manipulation in an appropriate power series ring. Two examples which do not are Ramanujan's ${ }_{1} \Psi_{1}[17]$ and the three term relations for ${ }_{3} \Phi_{2}$ 's [17]. 


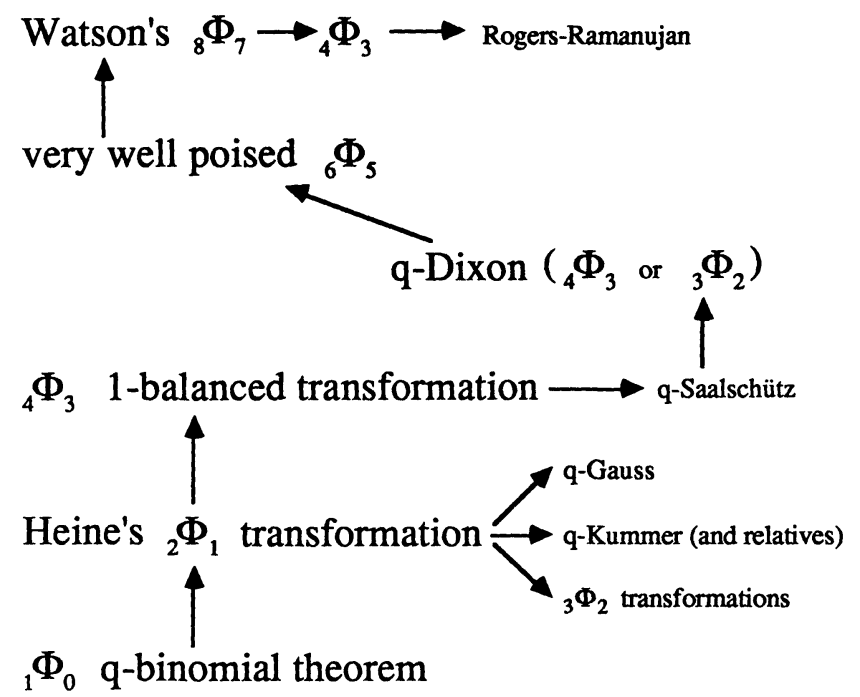

8. Remarks. While this paper has shown how to find a bijective proof of a basic hypergeometric identity, it is obviously not the final word on the subject. The emphasis should now be placed on combinatorially important proofs, rather than a proof. There will be several bijective proofs of a given identity because different bijections can be used for the $q$-binomial theorem. Also, different specializations ( say $a=q^{n}$ or $a=q^{-n}$ ) of an identity could have interesting yet completely different combinatorial interpretations. Finally, it would be interesting if our bijective proofs of the higher transformations could be explicitly identified.

It is ironic that the two ingredients of [1] which were explicitly missing-involutions and the Involution Principle-appeared implicitly. Instead of involutions, Andrews used inclusion-exclusion which he interpreted as an involution on "clumps" of elements. He also gave two such "involutions" and the match that would have resulted "in clumps" from the Involution Principle.

In [4] Andrews gave a bijective proof of the most difficult part of the Rogers-Ramanujan identities, the limiting case of the ${ }_{8} \Phi_{7}$ evaluation. The only other theorems which he used were the Jacobi triple product identity and a limiting case of the $q$-analogue of Gauss's theorem. These two theorems have bijective proof without the Involution Principle. His only non-bijective step was dividing by products then cancelling them.

\section{REFERENCES}

[1] G. Andrews, On a calculus of partition functions, Pacific J. Math., 31 (1969), 555-562. 
[3] _ On the q-analog of Kummer's theorem and applications, Duke Math. J., 40 (1973), 525-528.

[4] $\quad$ A new property of partitions with applications to the Rogers-Ramanujan identities, J. Combin. Theory-Ser. A, 10 (1971), 266-270.

[5] _ The Theory of Partitions, Addison-Wesley, Reading, Massachusetts, 1976.

[6] Connection coefficient problems and partitions, Proc. Symp. Pure Math., 34 (1979), 1-24.

[7] Multiple series Rogers-Ramanujan type identities, Pacific J. Math., 114 (1984), 267-283.

[8] _ Generalized Frobenius partitions, Mem. Amer. Math. Soc., 301 (1984).

[9] G. Andrews and D. M. Bressoud, Identities in combinatorics III: Further aspects of ordered set sorting, Discrete Math., 49 (1984), 222-236.

[10] P. Cartier and D. Foata, Problemes Combinatoires de Commutation et Réarrangements, Lecture Notes in Mathematics 85, Springer, Berlin, 1969.

[11] A. Garsia and S. Milne, Method for constructing bijections for classical partition identities, Proc. Nat. Acad. Sci. U.S.A., 78 (1981), 2026-2028.

[12] _ A Rogers-Ramanujan bijection, J. Combin. Theory-Ser. A, 31 (1981), 289-339.

[13] I. Gessel and G. Viennot, Binomial determinants, paths, and hook length formulae, Advances in Math., to appear.

[14] I. Goulden, A combinatorial proof of the q-Saalschütz identity, preprint.

[15] I. Goulden and D. Jackson, Combinatorial Enumeration, Wiley-Interscience, New York, 1983.

[16] J. Remmel, Bijective proofs of classical partition identities, J. Combin. Theory-Ser. A, 33 (1982), 273-286.

[17] L. Slater, Generalized Hypergeometric Functions, Cambridge University Press, Cambridge, 1966.

[18] J. J. Sylvester, A constructive theory of partitions, arranged in three acts, an interact and an exodion, Amer. J. Math. 5, 251-330; 6, 334-336 (or pp. 1-83 of The Collected Mathematical Papers of J. J. Sylvester, Vol. 4, Cambridge Univ. Press, London and New York, 1912; reprinted by Chelsea, New York, 1974).

[19] G. Viennot, Une théorie combinatoire des polynômes orthogonaux généraux, Lecture Notes, Université du Québec à Montréal, 1983.

[20] D. Zeilberger, A q-Foata proof of the q-Saalschütz identity, preprint.

Received September 3, 1985. The second author was partially supported by N.S.F. grant DMS: 8500958.

UNIVERSITY OF MINNESOTA

MiNNEAPOLIS, MN 55455 


\section{PACIFIC JOURNAL OF MATHEMATICS EDITORS}

\author{
V. S. VARADARAJAN \\ (Managing Editor) \\ University of California \\ Los Angeles, CA 90024 \\ Herbert Clemens \\ University of Utah \\ Salt Lake City, UT 84112 \\ R. FINN \\ Stanford University \\ Stanford, CA 94305
}

\author{
HERMANN FLASCHKA \\ University of Arizona \\ Tucson, AZ 85721 \\ RAMESh A. GANGOLli \\ University of Washington \\ Seattle, WA 98195 \\ VAUGHAN F. R. JONES \\ University of California \\ Berkeley, CA 94720 \\ ROBION KIRBY \\ University of California \\ Berkeley, CA 94720 \\ C. C. MOORE \\ University of California \\ Berkeley, CA 94720 \\ H. SAMELSON \\ Stanford University \\ Stanford, CA 94305 \\ HAROLD STARK \\ University of California, San Diego \\ La Jolla, CA 92093
}

\section{ASSOCIATE EDITORS}
R. ARENS
E. F. BECKENBACH
B. H. NEUMANN
F. WOLF
K. YOSHIDA (1906-1982)

\section{SUPPORTING INSTITUTIONS}
UNIVERSITY OF ARIZONA
UNIVERSITY OF BRITISH COLUMBIA
CALIFORNIA INSTITUTE OF TECHNOLOGY
UNIVERSITY OF CALIFORNIA
MONTANA STATE UNIVERSITY
UNIVERSITY OF NEVADA, RENO
NEW MEXICO STATE UNIVERSITY
OREGON STATE UNIVERSITY
UNIVERSITY OF OREGON UNIVERSITY OF SOUTHERN CALIFORNIA STANFORD UNIVERSITY UNIVERSITY OF HAWAII UNIVERSITY OF TOKYO UNIVERSITY OF UTAH WASHINGTON STATE UNIVERSITY UNIVERSITY OF WASHINGTON 


\section{Pacific Journal of Mathematics \\ Vol. 127, No. $1 \quad$ January, 1987}

Jacob Burbea, Boundary behavior of holomorphic functions in the ball ..... 1

Jan Dijkstra, Strong negligibility of $\sigma$-compacta does not characterize

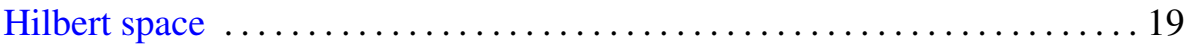

Ruy Exel, Rotation numbers for automorphisms of $C^{*}$ algebras $\ldots \ldots \ldots 31$

Howard Jacobowitz, The canonical bundle and realizable CR

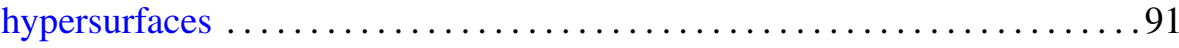

James T. Joichi and Dennis Warren Stanton, Bijective proofs of basic

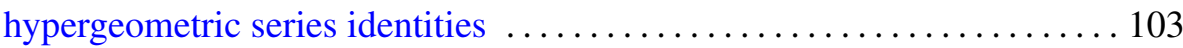

Gareth J. Knowles, Quotients of nest algebras with trivial commutator .... 121

Murray Angus Marshall, Exponentials and logarithms on Witt rings ..... 127

Courtney Hughes Moen, The dual pair $(U(3), U(1))$ over a $p$-adic field . ...141

William Ortmeyer, Surgery on a class of pretzel knots $\ldots \ldots \ldots \ldots \ldots \ldots 155$

John Gerard Ryan, Extensions of representations of Lie algebras . . . . . 173

Ivan Charles Sterling, A generalization of a theorem of Delaunay to

rotational $W$-hypersurfaces of $\sigma_{l}$-type in $H^{n+1}$ and $S^{n+1} \ldots \ldots \ldots 187$

Vesko M. Valov, Another characterization of AE(0)-spaces $\ldots \ldots \ldots \ldots \ldots 9$ 